\title{
Effects of isokinetic, isometric, and aerobic exercises on clinical variables and knee cartilage volume using magnetic resonance imaging in patients with osteoarthritis
}

\author{
Esin Benli Küçük, ${ }^{1}$ Özden Özyemişsi Taşkiran, ${ }^{2}$ Nil Tokgöz, ${ }^{3}$ Jale Meray ${ }^{4}$ \\ 'Department of Physical Medicine and Rehabilitation, Niğde Bor Physical Medicine and Rehabilitation Hospital, Niğde, Turkey \\ ${ }^{2}$ Department of Physical Medicine and Rehabilitation, Koç University School of Medicine, İstanbul, Turkey \\ ${ }^{3}$ Department of Radiology, Medical Faculty of Gazi University, Ankara, Turkey \\ ${ }^{4}$ Department of Physical Medicine and Rehabilitation, Medical Faculty of Gazi University, Ankara, Turkey \\ Received: September 07, 2016 Accepted: February 19, 2017 Published online: June 17, 2017
}

\begin{abstract}
Objectives: This study aims to evaluate the effect of isokinetic, isometric, and aerobic exercise protocols on pain, disability, physical function, and articular cartilage in osteoarthritis.

Patients and methods: A total of 45 women (mean age 52.1 years; range 45 to 65 years) who were admitted to the Physical Medicine and Rehabilitation outpatient clinic and were diagnosed with primary bilateral knee osteoarthritis between May 2008 and January 2010 were included. The patients were randomly divided into three groups as isokinetic $(n=15)$, aerobic ( $n=15)$, and isometric exercise groups $(\mathrm{n}=15)$. Exercise protocols were applied five days a week for four weeks. Pain was evaluated using a $10 \mathrm{~cm}$ Visual Analog Scale for Pain (VAS-pain), pain, joint stiffness and physical function was assessed using the Western Ontario and McMaster Universities Osteoarthritis Index (WOMAC), and disability was assessed using the Lequesne Index before and after the interventions. Isokinetic knee muscle strength measurements were also obtained. Patellar and femoral cartilage volumes were analyzed using magnetic resonance imaging.

Results: The VAS-pain, WOMAC, and Lequesne scores and peak torque values of knee extension improved in all groups with the highest improvement in the isokinetic group. For the knee flexion peak torque values, improvements were significant only in the isokinetic group at both velocities. There was no significant change in the femoral cartilage volume in any group after the interventions. However, patellar cartilage volume significantly increased in the isometric group $(\mathrm{p}=0.036)$.

Conclusion: A four-week isokinetic, aerobic, and isometric exercise programs improved pain and functional capacity in patients with knee osteoarthritis. Isokinetic exercise also increased the muscle strength with improved maintenance of the quadriceps/hamstring ratio. Only isometric exercise increased the patellar cartilage volume.
\end{abstract}

Keywords: Cartilage; exercise; knee osteoarthritis; magnetic resonance imaging.

Osteoarthritis (OA) is a common form of arthritis characterized by loss of articular cartilage. ${ }^{[1]}$ It is one of the major causes of physical disability and is the most common indication for hip and knee replacement. ${ }^{[2]}$ The main symptoms of $\mathrm{OA}$ are pain and loss of function. ${ }^{[3]}$

Exercise is a non-pharmacological treatment modality recommended in the guidelines of the management of knee OA. ${ }^{[3-5]}$ Although exercise reduces pain, improves physical function, aerobic capacity and endurance, and helps in weight reduction, there are still ongoing debates about the effects of exercise on the articular cartilage. ${ }^{[6,7]}$ Exercise may have a direct effect on the articular cartilage and an indirect effect through actions on muscles. Muscles play a key role in maintaining the normal biomechanics of the joint. Strengthening the quadriceps and hamstring muscles improves the joint stability. It has been shown that mechanical stimulation increases the biosynthetic activity of chondrocytes, and thickness of the cartilage adapts to mechanical loading in animal models. ${ }^{[8]}$ On the other hand, intense physical activity may increase the risk of OA. ${ }^{[9,10]}$

Corresponding author: Esin Benli Küçük, MD. Bor Fizik Tedavi Rehabilitasyon Hastanesi, Fiziksel Tıp ve Rehabilitasyon Kliniği, 51700 Bor, Niğde, Turkey. e-mail: esinbenli@yahoo.com.tr 
Magnetic resonance imaging (MRI) has enabled a direct evaluation of the articular cartilage. Using MRI, it is possible to assess the macro-morphological properties of the cartilage, i.e., thickness, surface area and volume of the articular cartilage. Surface irregularities of the cartilage and other structural changes in the joint can be also evaluated. ${ }^{[1]}$ Magnetic resonance imaging has multi-planar imaging capability, high spatial resolution without ionizing radiation, and superior contrast between the tissues of the joint. ${ }^{[12]}$ Therefore, it has gained popularity in the assessment of $\mathrm{OA}$ and the effects of exercise on the cartilage in recent years.

There are numerous studies investigating short-term effects of exercise on the cartilage. ${ }^{[13-18]}$ Deformation of the articular cartilage occurs shortly after exercise or physical activity ${ }^{[2]}$ and it takes about $90 \mathrm{~min}$ to recover after loading. ${ }^{[13]}$ During daily activities, patellar cartilage is subjected to 2 to $3 \%$ compression, compared to non-weightbearing states. ${ }^{[13]}$ Vigorous exercise adds another 2 to $3 \%$ compression over that of daily activities. ${ }^{[13]}$ Previously, deformation has been shown to be more prominent in the patellar cartilage, compared to the femoral and tibial cartilage. ${ }^{[13-17]}$

In the literature, studies addressing into the long-term effects of exercise on the cartilage are mostly cross-sectional, ${ }^{[1,19,20]}$ while the number of longitudinal studies has been also increasing in recent years. ${ }^{[21-23]}$ These studies, which primarily include healthy individuals without knee pain, have demonstrated that intense physical activity has beneficial effects on the articular cartilage of the knee ${ }^{[11,21]}$ and reduces cartilage loss in healthy adults. ${ }^{[22,23]}$ However, studies comparing the cartilage morphology of physically inactive subjects with that of athletes have shown that deformational behavior of the cartilage in the short-term after exercise is similar. ${ }^{[15,17]}$ In the long-term, the cartilage thickness is not different between athletes and physically inactive subjects, ${ }^{[19,20]}$ except for the study of Grtazke et al. ${ }^{[20]}$ who reported that only patellar cartilage was thicker in athletes.

In another study, Teichtahl et al. ${ }^{[23]}$ recruited healthy adults without clinical knee disease and found no relationship between vigorous physical activity and cartilage volume change or defect progression in the subgroup with prevalent patellar cartilage defects at baseline. However, the effects of exercise on the morphological features of articular cartilage in patients with knee OA have not been investigated using MRI, yet.
In the present study, we hypothesized that isokinetic and aerobic exercises would increase the muscle strength at the expense of reduction in the cartilage volume and both effects would be stronger after isokinetic exercises. Our second hypothesis was that isometric exercise would not alter the isokinetic muscle strength or cartilage morphology, but would reduce symptoms and increase the functional capacity. Therefore, we aimed to investigate the effects of isokinetic, isometric, and aerobic exercise protocols on the pain, functional capacity, muscle strength, and knee cartilage using MRI in patients with knee OA.

\section{PATIENTS AND METHODS}

This prospective, comparative study was approved by the Research Ethics Committee of Medical Faculty, Gazi University and conducted in accordance with the principles of the Declaration of Helsinki. A verbal and written informed consent was obtained from each participant.

A total of 45 women (mean age 52.1 years; range 45 to 65 years) who were admitted to the Physical Medicine and Rehabilitation outpatient clinic at a tertiary setting and were diagnosed with primary bilateral knee osteoarthritis according to the American College of Rheumatology criteria ${ }^{[24]}$ between May 2008 and January 2010 were included. All patients had radiological Grade 2 or 3 diseases, according to the Kellgren-Lawrence scale and were able to follow the exercise instructions. Patients with severe cardiac and pulmonary diseases, uncontrolled hypertension, inflammatory joint disease, prominent joint instability, previous knee surgery, lower extremity pathology other than knee OA, and those with an MRI contraindication were excluded from the study.

Eligible patients were randomly divided into three groups as isokinetic $(n=15)$, aerobic $(n=15)$, and isometric exercise groups $(n=15)$. A detailed history and physical examination findings including deformity, range of motion, swelling, tenderness, patellar mobility, crepitation, and ligament laxity in the knee joints were recorded. Age, height, weight, and body mass index were also noted.

Before and after interventions, self-reported pain severity during daily activities (i.e., walking, climbing stairs, and resting at night) was measured using the $10 \mathrm{~cm}$ Visual Analog Scale for Pain (VAS-pain). The Western Ontario and McMaster Universities Osteoarthritis Index (WOMAC) was used to evaluate 
pain, joint stiffness, and physical function. ${ }^{[25]}$ The Lequesne Index was used to assess disability.

The knee flexion and extension strength measurements were performed using the Cybex 770 Norm (Lumex Inc, Rankokoma, NY, USA) isokinetic dynamometer before and after the interventions. All measurements were performed, while the patient was seated with hip and knee joints flexed at $90^{\circ}$ and chest, trunk, and thigh proximal to the knee joint stabilized by straps. The lever arm was adjusted for the leg length of the patient and secured with a strap proximal to the ankle. The weight of the tested leg was measured before the test and the effect of gravity was adjusted by the dynamometer. Full extension of the knee joint was considered anatomical zero position. Tests were performed in a subject-specific range of motions, which were measured by the dynamometer. All measurements were performed before and after the interventions. Reciprocal concentric knee flexion and extension were evaluated at two angular velocities; $60 \%$ sec and $180 \%$ sec. Before testing, the patient was instructed about the each test and, with each angular velocity, four submaximal contractions were performed by the patient to become familiar with the tests. After the practice, the patient relaxed for a period of $10 \mathrm{sec}$ before test trials. During the measurements at $60 \% \mathrm{sec}$ angular velocity, the patient was asked to perform five repetitions of flexion and extension as forcefully as possible. Measurements at $180 \% \mathrm{sec}$ angular velocity were performed after resting for $20 \mathrm{sec}$. This time participants were asked to perform 20 repetitions of flexion and extension as soon as possible. The patient was, then, allowed to rest for $5 \mathrm{~min}$ between the measurements of the right and left knee. The peak torque values of the right knee recorded in foot-pounds (FtLbs) using the HUMAC software version 8.2.1 (Computer Sports Medicine, Inc., Stoughton, MA, USA) were used in the data analysis.

\section{Interventions}

All patients were instructed not to use nonsteroidal anti-inflammatory drugs during the study period. Only paracetamol was allowed, if needed, as an escape analgesic; however, it was not allowed to take paracetamol 24 hours before the assessment. Each participant performed the exercise protocols relevant to their group five days a week for four weeks in the orthopedic rehabilitation unit under the supervision of a research physiatrist (E.B.K.). All patients completed the exercise program relevant to their group and no patient was withdrawn from the study.
In the isokinetic group, the patients performed exercise using Cybex Norm Model 770 isokinetic dynamometer (Cybex, division of Lumex Inc., Ronkonkoma, NY, USA). After warming up, they performed 10 concentric-concentric flexion and extension contractions at $60^{\circ}, 90^{\circ}, 120^{\circ}, 150^{\circ}$ and $180^{\circ} / \mathrm{sec}$ angular velocity with $20 \mathrm{sec}$ rests rest between each angular velocity and five-min rest between the right and left knees. In the isometric group, the patients performed 10 straight leg raise and 10 quadriceps isometric contractions holding for $10 \mathrm{sec}$ with knee $90^{\circ}$ flexion and knee $180^{\circ}$ extension and a $2 \mathrm{~min}$ rest period between. In the aerobic group, the patients walked at $4.5 \mathrm{~km} /$ hour velocity on a treadmill for $20 \mathrm{~min}$.

After the interventions, transcutaneous electrical stimulation (TENS) and cold pack were applied to both knees of all patients in the three intervention groups for $20 \mathrm{~min}$.

A 1.5 Tesla MRI unit (Signa, Excite II, General Electric Medical Systems, Milwaukee, Wisconsin, USA) was used to assess the knee cartilage. Imaging of the knee was performed using a knee coil in the sagittal plane. A T-weighted fat-saturated spoiled gradient recalled acquisition in the steady state (SPGR) imaging sequence was performed in the sagittal plane using the following parameters: TR $40 \mathrm{~ms}$; TE $7 \mathrm{~ms}$; flip angle $40^{\circ}$; field of view $18 \times 15 \mathrm{~cm}$; matrix $288 \times 224$; number of excitations 2; slice thickness $1.5 \mathrm{~mm}$, inter-slice gap zero.

An independent workstation was used for the image analysis. During the analysis of MRI images, femoral and patellar articular cartilages were outlined manually using electronic cursors on a slice by slice basis. The volumes of the cartilages were derived automatically using commercially available software (Advantage Workstation, release 4.1, GE Healthcare, Waukesha, Wisconsin, USA). An experienced musculoskeletal radiologist performed the measurements on images within two days. To assess the intra- and inter-observer variations, the images of 15 patients were re-measured by the same observer and another radiologist in the following week.

The intra-class correlation coefficient (ICC) was used to evaluate the intra- and inter-observer correlations in two measurement sessions. The ICC indicated a high intra- and inter-observer reliability, as the value ranged from 0.83 to 0.91 for all measurements.

\section{Statistical analysis}

Statistical analysis was performed using the SPSS for Windows version 15.0 (SPSS Inc., Chicago, IL, USA). 
Table 1. Demographic and clinical characteristics of the participants

\begin{tabular}{|c|c|c|c|c|c|c|c|c|c|c|}
\hline & \multicolumn{3}{|c|}{ Isokinetic group $(\mathrm{n}=15)$} & \multicolumn{3}{|c|}{ Aerobic group $(\mathrm{n}=15)$} & \multicolumn{3}{|c|}{ Isometric group $(\mathrm{n}=15)$} & \multirow[b]{2}{*}{$p$} \\
\hline & $\mathrm{n}$ & $\%$ & Mean \pm SD & $\mathrm{n}$ & $\%$ & Mean \pm SD & $\mathrm{n}$ & $\%$ & Mean \pm SD & \\
\hline Age (year) & & & $51.5 \pm 5.0$ & & & $52.5 \pm 5.3$ & & & $52.3 \pm 6.9$ & 0.863 \\
\hline Body weight (kg) & & & $74.9 \pm 12.8$ & & & $74.1 \pm 12.6$ & & & $71.9 \pm 14.8$ & 0.762 \\
\hline Body height (cm) & & & $157.7 \pm 4.5$ & & & $157.7 \pm 7.8$ & & & $155.6 \pm 7.5$ & 0.768 \\
\hline Body mass index $\left(\mathrm{kg} / \mathrm{cm}^{2}\right)$ & & & $30.1 \pm 5.0$ & & & $29.6 \pm 3.5$ & & & $29.9 \pm 7.2$ & 0.980 \\
\hline $\begin{array}{l}\text { Radiographic Grade of right knee } \\
\text { (Kellgren-Lawrence) }\end{array}$ & & & & & & & & & & 0.435 \\
\hline Grade 2 & 8 & 53 & & 11 & 73 & & 8 & 53 & & \\
\hline Grade 3 & 7 & 47 & & 4 & 27 & & 7 & 47 & & \\
\hline
\end{tabular}

SD: Standard deviation.

The Shapiro-Wilk test was used to test the normality of distribution of each of the variable. Categorical variables were expressed in median and/or mean \pm standard deviation (SD) for quantitative variables and proportions (\%). The Kruskal-Wallis test was used to test the significant differences in age, weight, height, body mass index, and also the baseline measurements of VAS, WOMAC scores, Lequesne Index, peak torque measurements and femoral and patellar cartilage volumes among the groups. The Wilcoxon signed-rank test was used to compare preand post-exercise values within the same group for VAS, VOMAC scores, Lequesne Index, peak torque measurements and femoral and patellar cartilage volumes. Two-way mixed analysis of variance (ANOVA) was used to compare the effect of exercise programs on VAS, WOMAC scores, Lequesne Index, peak torque measurements, and femoral and patellar cartilage volumes among the groups. The chi-square test was used to compare the radiographic Grades of the right knee (Kellgren-Lawrence) among the groups. A $p$ value of $<0.05$ was considered statistically significant.

\section{RESULTS}

Forty-five women with bilateral knee OA completed the study. There were no significant differences in age, weight, height, body mass index among the groups. Although a higher proportion of patients in the aerobic group was Grade 2 according to the Kellgren-Lawrence grading scale, this difference was not statistically significant among the exercise groups $(\mathrm{p}=0.435)$ (Table 1$)$.

Baseline values of outcome parameters are presented in Table 2. There were no significant differences in the VAS-scores during the night, climbing stairs or walking; WOMAC subscores of pain, stiffness and

Table 2. Pain scores according to VAS, WOMAC, and Lequesne Index before and after interventions

\begin{tabular}{|c|c|c|c|c|c|c|c|}
\hline & \multicolumn{2}{|c|}{ Isokinetic group $(\mathrm{n}=15)$} & \multicolumn{2}{|c|}{ Aerobic group $(n=15)$} & \multicolumn{2}{|c|}{ Isometric group $(n=15)$} & \multirow[b]{2}{*}{$p$} \\
\hline & Median & Min-Max & Median & Min-Max & Median & Min-Max & \\
\hline \multirow[t]{2}{*}{ VAS-walking $(\mathrm{cm})$} & 7.0 & $4.0-9.0$ & 7.0 & $5.0-9.0$ & 7.0 & $5.0-9.0$ & \multirow{2}{*}{0.776} \\
\hline & 4.0 & $2.0-9.0^{* *}$ & 4.0 & $2.0-6.0^{* *}$ & 4.0 & $2.0-5.0^{\star *}$ & \\
\hline \multirow[t]{2}{*}{ VAS-night (cm) } & 5.0 & $1.0-8.0$ & 2.0 & $1.0-7.0$ & 4.0 & $1.0-7.0$ & \multirow{2}{*}{0.781} \\
\hline & 2.0 & $1.0-4.0^{\star *}$ & 2.0 & $1.0-5.0^{\star}$ & 2.0 & $1.0-4.0^{\star \star}$ & \\
\hline \multirow[t]{2}{*}{ VAS-climbing $(\mathrm{cm})$} & 8.0 & $6.0-10.0$ & 7.0 & $5.0-9.0$ & 7.0 & $6.0-9.0$ & \multirow{2}{*}{0.956} \\
\hline & 4.0 & $2.0-8.0^{\star *}$ & 5.0 & $4.0-6.0^{* *}$ & 4.0 & $3.0-6.0^{\star *}$ & \\
\hline \multirow[t]{2}{*}{ WOMAC-pain } & 14.0 & $11.0-20.0$ & 15.0 & $10.0-19.0$ & 15.0 & $9.0-19.0$ & \multirow{2}{*}{0.933} \\
\hline & 8.0 & $5.0-18.0^{* *}$ & 8.0 & $7.0-12.0^{\star *}$ & 7.0 & $5.0-12.0^{\star *}$ & \\
\hline \multirow[t]{2}{*}{ WOMAC-stiffness } & 5.0 & $2.0-8.0$ & 6.0 & $2.0-8.0$ & 6.0 & $2.0-8.0$ & \multirow{2}{*}{0.490} \\
\hline & 3.0 & $2.0-8.0^{*}$ & 3.0 & $2.0-6.0^{\star *}$ & 4.0 & $2.0-6.0^{\star *}$ & \\
\hline \multirow[t]{2}{*}{ WOMAC-function } & 43.0 & $32.0-60.0$ & 45.0 & $34.0-71.0$ & 48.0 & $28.0-66.0$ & \multirow{2}{*}{0.990} \\
\hline & 25.0 & $20.0-68.0^{* *}$ & 25.0 & $21.0-47.0^{* *}$ & 30.0 & $20.0-38.0^{* *}$ & \\
\hline \multirow[t]{2}{*}{ WOMAC-total } & 62.0 & $50.0-83.0$ & 63.0 & $50.0-96.0$ & 72.0 & $39.0-92.0$ & \multirow[t]{2}{*}{0.975} \\
\hline & 37.0 & $28.0-94.0^{* *}$ & 35.0 & $31.0-65.0^{* *}$ & 42.0 & $27.0-50.0^{* *}$ & \\
\hline \multirow[t]{2}{*}{ Lequesne Index } & 11.0 & $7.0-18.0$ & 11.0 & $6.0-15.0$ & 10.0 & $6.0-15.0$ & \multirow{2}{*}{0.519} \\
\hline & 6.0 & $1.0-13 \cdot 0^{* *}$ & 6.0 & $3.0-11.0^{\star \star}$ & 5.0 & $3.0-8.0^{\star *}$ & \\
\hline
\end{tabular}

VAS: Visual Analog Scale; WOMAC: Western Ontario and McMaster Universities Osteoarthritis Index; Min: Minimum; Max: Maximum; ${ }^{\star} \mathrm{p} \leq 0.05 ;{ }^{\star *} \mathrm{p} \leq 0.01$; difference after intervention within the same group; The results before the interventions are depicted in the first line and the results after the interventions are depicted in the second line. $P$ values are defined for the comparison of differences (before and after interventions) among the groups. 
(a)

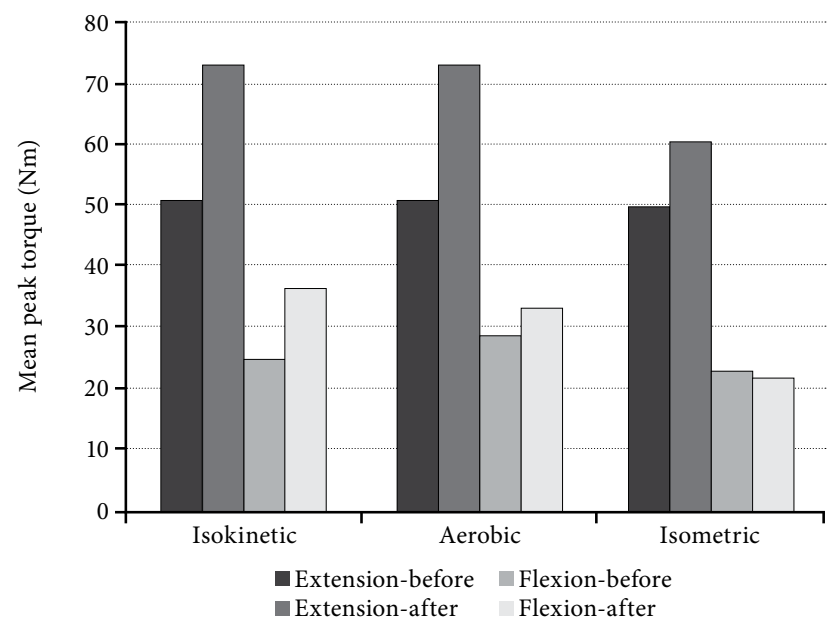

(b)

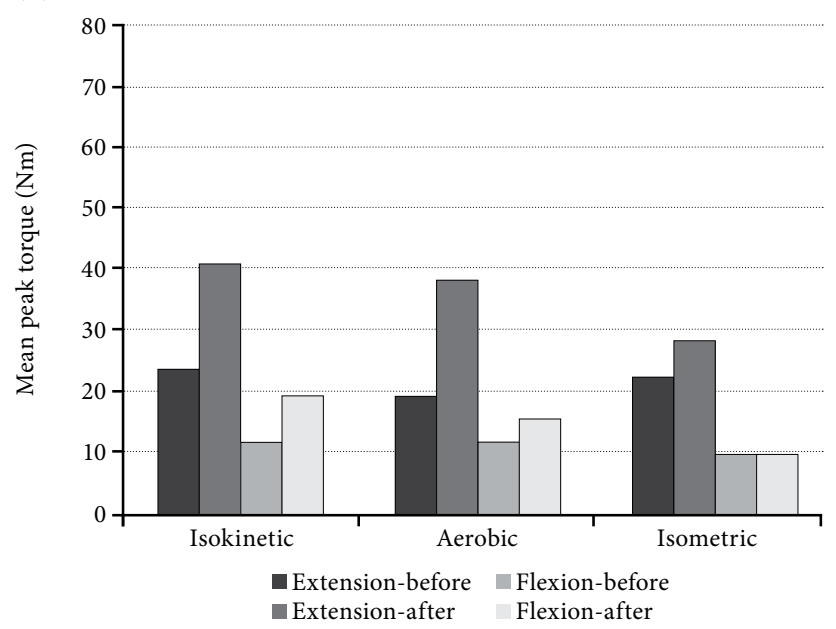

Figure 1. Peak torque measurements of the right knee flexion and extension at (a) $60 \% \mathrm{sec}$ and (b) $180 \%$ sec angular velocity before and after interventions among groups.

function or total scores; Lequesne disability scores; peak torque values of knee flexion or extension measured at $60^{\circ}$ and $180^{\circ} / \mathrm{sec}$ angular velocities; patellar or femoral cartilage volumes before the interventions among the three groups.

Significant improvements in pain scores during daily activities (i.e., walking, climbing up and down stairs, and night pain) were observed in all groups after the interventions, although the difference did not reach statistical significance (Table 2).

The peak torque values of the knee flexion and extension measured at $60^{\circ}$ and $180^{\circ} / \mathrm{sec}$ angular velocities of the right knees in all groups are shown in Figure 1a, b. In the isokinetic group, the peak torque values of the knee flexion and extension at $60^{\circ}$ and $180 \% \mathrm{sec}$ angular velocities improved significantly after four-week training $(\mathrm{p}=0.003$ and $\mathrm{p}=0.007$ for $60 \% \mathrm{sec}$; $\mathrm{p}=0.009$ and $\mathrm{p}=0.004$ for $180 \% \mathrm{sec}$, respectively). In the aerobic group, the peak torque improvements in knee extension were statistically significant $(\mathrm{p}=0.008$ for $60 \% \mathrm{sec} ; \mathrm{p}=0.009$ for $180^{\circ} \mathrm{sec}$ ), whereas those of the knee flexion were not $(p=0.293$ and $p=0.148)$. In the isometric group, the peak torques of knee extension at both velocities increased significantly ( $\mathrm{p}=0.025$ for $60 \% \mathrm{sec} ; \mathrm{p}=0.041$ for $180 \% \mathrm{sec}$ ). However, the flexion peak torques did not change significantly $(p=0.683$ and $\mathrm{p}=0.864$ ).

The improvements in the knee flexion peak torque at $60 \% \mathrm{sec}$ angular velocity were not significantly different among groups $(\mathrm{p}=0.094)$. Also, the improvements in knee flexion peak torque at $180 \% \mathrm{sec}$ angular velocity did not reach statistical significance $(p=0.071)$. The changes in the peak torques were not different significantly for knee extension at $60 \% \mathrm{sec}$ or $180 \%$ sec angular velocities $(\mathrm{p}=0.522$ and $\mathrm{p}=0.344$, respectively) among the groups.

The hamstring/quadriceps ratio remained nearly unchanged in the isokinetic group and decreased in the aerobic and isometric groups $(\mathrm{p}=0.172$ and $\mathrm{p}=0.017$, respectively); however, only this ratio was significant in the isometric group.

The femoral and cartilage volumes of right knees before and after the interventions are shown in Figure 2. No significant change in femoral cartilage volume was observed in any of the groups after the interventions (Figure 2, Table 3). Regarding patellar cartilage volume, the mean difference was found

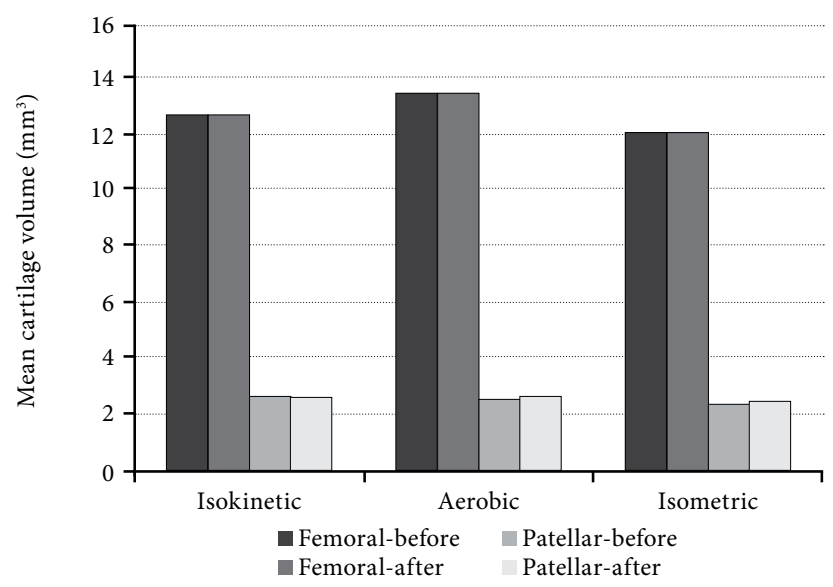

Figure 2. Femoral and patellar cartilage volumes before and after interventions. 
to be statistically significant in the isometric group $\left(2.24 \pm 0.29\right.$ and $2.35 \pm 0.34 \mathrm{~mm}^{3}$ before and after intervention, respectively, $\mathrm{p}=0.036$ ), whereas in the isokinetic and aerobic groups the changes did not significantly differ ( $\mathrm{p}=0.533$ and $\mathrm{p}=0.327$, respectively) (Table 3). Also, there was no significant difference among the three exercise groups $(\mathrm{p}=0.338)$.

\section{DISCUSSION}

Isokinetic, aerobic, and isometric training for four weeks improved all clinical outcome variables including pain which was assessed according to the VAS, WOMAC, and Lequesne Index. Regarding the isokinetic strength measurements, the peak torque values of knee extension at $60^{\circ}$ and $180^{\circ} / \mathrm{sec}$ angular velocities increased in all groups, and the highest change was in the isokinetic group. For the knee flexion peak torque values, improvements were significant only in the isokinetic group at both angular velocities. Morphological measurements of femoral and patellar cartilage using MRI revealed that the reductions observed after isokinetic training were not prominent enough to reach statistical significance. In the aerobic group, increments in both cartilage volumes were observed, rather than declines, although no statistically significance was reached. Interestingly, patellar cartilage volumes improved significantly in the isometric group, while the femoral cartilage volumes remained unchanged.

Our first hypothesis that isokinetic and aerobic exercises would increase the muscle strength at the expense of reduction in cartilage volume and both effects would be stronger after isokinetic exercise" proved to be partially true. The flexion peak torques did not increase significantly in the aerobic group, as anticipated, but the expected reductions in cartilage volumes were unable to be achieved. Our second hypothesis that "isometric exercise would not alter the isokinetic muscle strength or cartilage morphology, but would reduce symptoms and increase the functional capacity" was unable to be fully met. Although isometric exercises improved the clinical outcome measures, unexpectedly, it also improved the extensor peak torque and patellar cartilage volume.

Currently, in the management of knee OA, complete cure of the disease cannot be achieved; however, modification of the disease is possible. Recommendations for the management of knee OA aim at reducing pain and physical disability, while limiting the structural joint deterioration. ${ }^{[26]}$ Exercise is one of the most important non-pharmacological 
treatment options which decrease pain and improve physical functions and is recommended in all of the guidelines developed for management of knee OA. ${ }^{[3-5,26]}$ Although aerobic and resistance exercises have been shown to be beneficial, the optimal exercise type and frequency have not been clearly defined, yet. ${ }^{[4,5,26,27]}$

The quadriceps muscle has an important stabilizing role in maintaining the normal biomechanics of the knee. Weakness of the quadriceps muscle is common in knee OA due to disuse atrophy secondary to joint pain. On the other hand, quadriceps weakness is thought to be a risk factor for the progression of joint damage. ${ }^{[28-30]}$ Isometric and isokinetic muscle strength measurements of the knee extension and also flexion in patients with knee OA have been shown to be lower than in healthy controls. ${ }^{[31]}$ Strengthening exercises of the quadriceps and hamstring muscles may improve the joint stability.

Huang et al. ${ }^{[32]}$ reported that isometric, isotonic, and isokinetic exercises decreased pain and disability, while increasing walking speed in patients with knee OA. The greatest improvement in walking speed, muscle strength, and physical function was observed after isokinetic exercises. However, Eyigor ${ }^{[33]}$ demonstrated no superiority of isokinetic exercise over isotonic exercise in terms of walking speed and muscle strength. In our study, isokinetic exercise improved both flexor and extensor muscle strengths, whereas isometric and aerobic exercises improved the extensor muscle strength, but not the flexor muscle strength. Based on these findings, we suggest that isokinetic exercises may provide more benefits through effective stabilization with improvements in both agonistantagonist muscle groups.

Knowledge about the effects of different types of exercise on cartilage morphology is important in the formulation of optimal exercise prescription in OA. Wijayaretne et al. ${ }^{[22]}$ investigated the rate of annual patellar cartilage volume loss and its determinants in healthy middle-aged woman. They found that the annual loss of patellar cartilage volume was $1.6 \%$ in their patient group and participation in exercise tended to decrease rate of cartilage loss, but the type of exercise was not specified. Teichtahl et al. ${ }^{[23]}$ also investigated the effect of vigorous physical activity on the patellar cartilage volume using MRI. After a two-year follow-up period, they found that vigorous physical activity reduced annual rate of the patellar cartilage volume loss particularly in healthy adults without any cartilage defects. They, therefore, concluded that weight-bearing vigorous physical activity might be helpful in the prevention of knee OA. In both studies, the data regarding the exercise were obtained from the patients using a questionnaire.

In another study, Racunica et al. ${ }^{[1]}$ investigated the effect of physical activity on the knee structures of healthy adults using MRI. They found that walking had a beneficial effect on the reduction of bone marrow lesions in the knee, but that had no effect on the tibial cartilage volume. The authors reported that previous or recent vigorous physical activity was associated with an increased tibial cartilage volume. Based on these findings, they concluded that vigorous physical activity had a protective effect on the knee cartilage. However, the amount of physical activity to produce this effect was not clearly specified. Similarly, data regarding the exercise were obtained using a questionnaire.

In addition, Gratzke et al. ${ }^{[20]}$ found no significant difference in the cartilage thickness, except for the patellar cartilage in athletes, compared to the physically inactive participants. Weightlifters had a thicker patellar cartilage than untrained healthy individuals. Unique to all these studies, participants were healthy without any knee problems and they were evaluated based on preferred lifestyle, either physically active or inactive.

Cotofana et al. ${ }^{[34]}$ also investigated the knee joint morphology after 12 weeks of endurance (cycling) and strength training to prove protection against knee $\mathrm{OA}$ in untrained middle-aged women without $\mathrm{OA}$. They found no significant alterations in the cartilage thickness or volume using MRI. However, they showed a temporal relationship between exercise and cartilage morphology, although none of the participants were OA patients.

In a cross-sectional study conducted by Creaby et al., ${ }^{[35]}$ mechanical loading during walking might have a role in the pathological alterations in the articular cartilage in patients with OA. Different types of exercise result in different mechanical loading on the cartilage. In our study, four-week isokinetic, isometric, and aerobic exercise interventions did not have an unfavorable effect on the patellar or femoral articular cartilage, and isometric exercises even increased the patellar cartilage volume.

The main strength of the present study is that it is the first longitudinal study investigating the effects of different types of exercise on the cartilage morphology in patients with OA. A further strength of our study is 
that all of the training sessions were performed under the supervision of a physiatrist. A potential strength of our study is the availability of an isokinetic exercise group, compared to the isometric and aerobic groups.

On the other hand, there are some limitations to this study. Longer duration of training interventions and the presence of a control group with no exercise intervention would strengthen our results. A second limitation was that tibial cartilage volume could not be measured and volumes of entire cartilage were measured without dividing into medial and lateral regions due to technical difficulties. These might lead to the underestimation of focal volume changes in case of non-uniform regional variations. The study is also limited by the inclusion only of women with OA; therefore, the results cannot be generalized to men or healthy adults.

In conclusion, isokinetic, aerobic, and isometric training for four weeks improved pain and functional capacity in patients with knee OA, without superiority of one type of exercise to another. Isokinetic exercise led to increments in the muscle strength measurements with improved maintenance of the quadriceps/hamstring ratio. We observed no significant unfavorable effect of any exercise type on the femoral or patellar cartilage volume. Only isometric exercise increased the patellar cartilage volume.

\section{Declaration of conflicting interests}

The authors declared no conflicts of interest with respect to the authorship and/or publication of this article.

\section{Funding}

The costs of radiological assessments were funded by the Scientific Research Project Unit of Gazi University.

\section{REFERENCES}

1. Lawrence RC, Felson DT, Helmick CG, Arnold LM, Choi $\mathrm{H}$, Deyo RA, et al. Estimates of the prevalence of arthritis and other rheumatic conditions in the United States. Part II. Arthritis Rheum 2008;58:26-35.

2. Zhang Y, Jordan JM. Epidemiology of osteoarthritis. Clin Geriatr Med 2010;26:355-69.

3. Recommendations for the medical management of osteoarthritis of the hip and knee: 2000 update. American College of Rheumatology Subcommittee on Osteoarthritis Guidelines. Arthritis Rheum 2000;43:1905-15.

4. Jordan KM, Arden NK, Doherty M, Bannwarth B, Bijlsma JW, Dieppe P, et al. EULAR Recommendations 2003: an evidence based approach to the management of knee osteoarthritis: Report of a Task Force of the Standing Committee for International Clinical Studies Including Therapeutic Trials (ESCISIT). Ann Rheum Dis 2003;62:1145-55.
5. Zhang W, Moskowitz RW, Nuki G, Abramson S, Altman $\mathrm{RD}$, Arden N, et al. OARSI recommendations for the management of hip and knee osteoarthritis, Part II: OARSI evidence-based, expert consensus guidelines. Osteoarthritis Cartilage 2008;16:137-62.

6. Jamtvedt G, Dahm KT, Christie A, Moe RH, Haavardsholm E, Holm I, et al. Physical therapy interventions for patients with osteoarthritis of the knee: an overview of systematic reviews. Phys Ther 2008;88:123-36.

7. Hunter DJ, Lo GH. The management of osteoarthritis: an overview and call to appropriate conservative treatment. Rheum Dis Clin North Am 2008;34:689-712.

8. Jin M, Frank EH, Quinn TM, Hunziker EB, Grodzinsky AJ. Tissue shear deformation stimulates proteoglycan and protein biosynthesis in bovine cartilage explants. Arch Biochem Biophys 2001;395:41-8.

9. Lequesne MG, Dang N, Lane NE. Sport practice and osteoarthritis of the limbs. Osteoarthritis Cartilage 1997;5:75-86.

10. Spector TD, Harris PA, Hart DJ, Cicuttini FM, Nandra D, Etherington J, et al. Risk of osteoarthritis associated with long-term weight-bearing sports: a radiologic survey of the hips and knees in female ex-athletes and population controls. Arthritis Rheum 1996;39:988-95.

11. Racunica TL, Teichtahl AJ, Wang Y, Wluka AE, English DR, Giles GG, et al. Effect of physical activity on articular knee joint structures in community-based adults. Arthritis Rheum 2007;57:1261-8.

12. Blumenkrantz G, Majumdar S. Quantitative magnetic resonance imaging of articular cartilage in osteoarthritis. Eur Cell Mater 2007;13:76-86.

13. Eckstein F, Hudelmaier M, Putz R. The effects of exercise on human articular cartilage. J Anat 2006;208:491-512.

14. Eckstein F, Tieschky M, Faber S, Englmeier KH, Reiser M. Functional analysis of articular cartilage deformation, recovery, and fluid flow following dynamic exercise in vivo. Anat Embryol (Berl) 1999;200:419-24.

15. Mosher TJ, Liu Y, Torok CM. Functional cartilage MRI T2 mapping: evaluating the effect of age and training on knee cartilage response to running. Osteoarthritis Cartilage 2010;18:358-64.

16. Boocock M, McNair P, Cicuttini F, Stuart A, Sinclair T. The short-term effects of running on the deformation of knee articular cartilage and its relationship to biomechanical loads at the knee. Osteoarthritis Cartilage 2009;17:883-90.

17. Eckstein F, Lemberger B, Gratzke C, Hudelmaier M, Glaser $\mathrm{C}$, Englmeier $\mathrm{KH}$, et al. In vivo cartilage deformation after different types of activity and its dependence on physical training status. Ann Rheum Dis 2005;64:291-5.

18. Kersting UG, Stubendorff JJ, Schmidt MC, Brüggemann GP. Changes in knee cartilage volume and serum COMP concentration after running exercise. Osteoarthritis Cartilage 2005;13:925-34.

19. Eckstein F, Faber S, Mühlbauer R, Hohe J, Englmeier KH, Reiser M, et al. Functional adaptation of human joints to mechanical stimuli. Osteoarthritis Cartilage 2002;10:44-50.

20. Gratzke C, Hudelmaier M, Hitzl W, Glaser C, Eckstein F. Knee cartilage morphologic characteristics and muscle status of professional weight lifters and sprinters: a magnetic resonance imaging study. Am J Sports Med 2007;35:1346-53. 
21. Foley S, Ding C, Cicuttini F, Jones G. Physical activity and knee structural change: a longitudinal study using MRI. Med Sci Sports Exerc 2007;39:426-34.

22. Wijayaratne SP, Teichtahl AJ, Wluka AE, Hanna F, Bell $\mathrm{R}$, Davis SR, et al. The determinants of change in patella cartilage volume--a cohort study of healthy middle-aged women. Rheumatology (Oxford) 2008;47:1426-9.

23. Teichtahl AJ, Wluka AE, Forbes A, Wang Y, English DR, Giles GG, et al. Longitudinal effect of vigorous physical activity on patella cartilage morphology in people without clinical knee disease. Arthritis Rheum 2009;61:1095-102.

24. Altman R, Asch E, Bloch D, Bole G, Borenstein D, Brandt $K$, et al. Development of criteria for the classification and reporting of osteoarthritis. Classification of osteoarthritis of the knee. Diagnostic and Therapeutic Criteria Committee of the American Rheumatism Association. Arthritis Rheum 1986;29:1039-49.

25. Wolfe F, Kong SX. Rasch analysis of the Western Ontario MacMaster questionnaire (WOMAC) in 2205 patients with osteoarthritis, rheumatoid arthritis, and fibromyalgia. Ann Rheum Dis 1999;58:563-8.

26. Zhang W, Moskowitz RW, Nuki G, Abramson S, Altman $\mathrm{RD}$, Arden $\mathrm{N}$, et al. OARSI recommendations for the management of hip and knee osteoarthritis, part I: critical appraisal of existing treatment guidelines and systematic review of current research evidence. Osteoarthritis Cartilage 2007;15:981-1000.

27. Roddy E, Zhang W, Doherty M, Arden NK, Barlow J, Birrell F, et al. Evidence-based recommendations for the role of exercise in the management of osteoarthritis of the hip or knee--the MOVE consensus. Rheumatology (Oxford) 2005;44:67-73.
28. Slemenda C, Brandt KD, Heilman DK, Mazzuca S, Braunstein EM, Katz BP, et al. Quadriceps weakness and osteoarthritis of the knee. Ann Intern Med 1997;127:97-104.

29. Slemenda C, Heilman DK, Brandt KD, Katz BP, Mazzuca SA, Braunstein EM, et al. Reduced quadriceps strength relative to body weight: a risk factor for knee osteoarthritis in women? Arthritis Rheum 1998;41:1951-9.

30. Brandt KD, Heilman DK, Slemenda C, Katz BP, Mazzuca SA, Braunstein EM, et al. Quadriceps strength in women with radiographically progressive osteoarthritis of the knee and those with stable radiographic changes. J Rheumatol 1999;26:2431-7.

31. Tan J, Balci N, Sepici V, Gener FA. Isokinetic and isometric strength in osteoarthrosis of the knee. A comparative study with healthy women. Am J Phys Med Rehabil 1995;74:364-9.

32. Huang MH, Lin YS, Yang RC, Lee CL. A comparison of various therapeutic exercises on the functional status of patients with knee osteoarthritis. Semin Arthritis Rheum 2003;32:398-406.

33. Eyigor S, Hepguler S, Capaci K. A comparison of muscle training methods in patients with knee osteoarthritis. Clin Rheumatol 2004;23:109-15.

34. Cotofana S, Ring-Dimitriou S, Hudelmaier M, Himmer M, Wirth W, Sänger AM, et al. Effects of exercise intervention on knee morphology in middle-aged women: a longitudinal analysis using magnetic resonance imaging. Cells Tissues Organs 2010;192:64-72.

35. Creaby MW, Wang Y, Bennell KL, Hinman RS, Metcalf $\mathrm{BR}$, Bowles KA, et al. Dynamic knee loading is related to cartilage defects and tibial plateau bone area in medial knee osteoarthritis. Osteoarthritis Cartilage 2010;18:1380-5. 